\title{
Persistence and Nonpersistence of a Predator Prey System with Stochastic Perturbation
}

\author{
Haihong Li, ${ }^{1,2}$ Daqing Jiang, ${ }^{1}$ Fuzhong Cong, ${ }^{2,3}$ and Haixia $\mathrm{Li}^{4}$ \\ ${ }^{1}$ College of Science, China University of Petroleum (East China), Qingdao 266580, China \\ ${ }^{2}$ Department of Basic Courses, Air Force Aviation University, Changchun, Jilin 130022, China \\ ${ }^{3}$ School of Mathematics, Jilin University, Changchun, Jilin 130024, China \\ ${ }^{4}$ School of Business, Northeast Normal University, Changchun, Jilin 130024, China \\ Correspondence should be addressed to Daqing Jiang; daqingjiang2010@hotmail.com
}

Received 13 December 2013; Accepted 25 February 2014; Published 3 April 2014

Academic Editor: Jifeng Chu

Copyright (c) 2014 Haihong Li et al. This is an open access article distributed under the Creative Commons Attribution License, which permits unrestricted use, distribution, and reproduction in any medium, provided the original work is properly cited.

\begin{abstract}
We analyze a predator prey model with stochastic perturbation. First, we show that this system has a unique positive solution. Then, we deduce conditions that the system is persistent in time average. Furthermore, we show the conditions that there is a stationary distribution of the system which implies that the system is permanent. After that, conditions for the system going extinct in probability are established. At last, numerical simulations are carried out to support our results.
\end{abstract}

\section{Introduction}

Recently, the dynamic relationship between predator and prey has been one of the dominant themes in both ecology and mathematical ecology due to its universal importance. Especially, the predator prey model is the typical representative. Thereby it significantly changed the biology and the understanding of the existence and development of the basic law and has made the model become the research hot spot. One of the most famous models for population dynamics is the Lotka-Volterra predator prey system which has received plenty of attention and has been studied extensively; we refer the reader to [1-3] for details. Specially persistence and extinction of this model are interesting topics.

The predator prey model is described as follows:

$$
\begin{gathered}
\dot{x}(t)=r x(t)\left(1-\frac{x(t)}{K}\right)-c x(t) y(t), \\
\dot{y}(t)=-\mu y(t)+m c x(t) y(t),
\end{gathered}
$$

where $x(t), y(t)$ denote the population densities of the species at time $t$. The parameters $r, K, c, \mu, m$, are positive constants that stand for prey intrinsic growth rate, carrying capacity, the maximum ingestion rate, predator death rate, and the conversion factor, respectively. From a biological viewpoint, we not only require the positive solution of the system but also require its unexploded property in any finite time and stability. We know that system (1) has a unique positive equilibrium $\left(x^{*}, y^{*}\right)$ which is a stable node or focus if the following condition holds, $m c K>\mu$ :

$$
x^{*}=\frac{\mu}{m c}, \quad y^{*}=\frac{r m c K-r \mu}{m c^{2} K}
$$

and the system (1) has a unique limit cycle which is stable (see [4]).

However, population dynamics in the real world is inevitably affected by environmental noise (see, e.g., [5-7]). Parameters involved in the system are not absolute constants; they always fluctuate around some average values. The deterministic models assume that parameters in the systems are deterministic irrespective of environmental fluctuations which impose some limitations in mathematical modeling of ecological systems. So we cannot omit the influence of the noise on the system. Recently many authors have discussed population systems subject to white noise (see, e.g., [8-12]). May (see, e.g., [13]) pointed out that due to continuous 
fluctuation in the environment, the birth rates, death rates, saturated rate, competition coefficients, and all other parameters involved in the model exhibit random fluctuation to some extent, and as a result the equilibrium population distribution never attains a steady value but fluctuates randomly around some average value. Sometimes, large amplitude fluctuation in population will lead to the extinction of certain species, which does not happen in deterministic models.

Therefore, Lotka-Volterra predator prey models in random environments are becoming more and more popular. Ji et al. [14, 15] investigated the asymptotic behavior of the stochastic predator prey system with perturbation. Liu and Chen [16] introduced periodic constant impulsive immigration of predator into predator prey system and gave conditions for the system to be extinct and permanent.

In this paper, we introduce the white noise into the intrinsic growth rate and predator death rate of system (1); that is, $r \rightarrow r+\sigma_{1} \dot{B}_{1}(t), \mu \rightarrow \mu+\sigma_{2} \dot{B}_{2}(t)$; then, we obtain the following stochastic system:

$$
\begin{gathered}
\dot{x}(t)=r x(t)\left(1-\frac{x(t)}{K}\right)-c x(t) y(t) \\
+\frac{\sigma_{1}}{K} x(t)(K-x(t)) \dot{B}_{1}(t), \\
\dot{y}(t)=-\mu y(t)+m c x(t) y(t)-\sigma_{2} y(t) \dot{B}_{2}(t),
\end{gathered}
$$

where $B_{i}(t)(i=1,2)$ are independent white noises with $B_{i}(0)=0, \sigma_{i}^{2}>0(i=1,2)$ representing the intensities of the noise.

The aim of this paper is to discuss the long time behavior of system (3). We have mentioned that $\left(x^{*}, y^{*}\right)$ is the positive equilibrium of system (1). But when it suffers stochastic perturbations, there is no positive equilibrium. Hence, it is impossible that the solution of system (3) will tend to a fixed point. In this paper, we show that system (3) is persistent in time average. Furthermore, under certain conditions, we prove that the population of system (3) will die out in probability which will not happen in deterministic system and could reveal that large white noise may lead to extinction.

The rest of this paper is organized as follows. In Section 2, we show that there is a unique nonnegative solution of system (3). In Section 3, we show that system (3) is persistent in time average, while in Section 4 we consider three situations when the population of the system will be extinct. In Section 5, numerical simulations are carried out to support our results.

Throughout this paper, unless otherwise specified, let $\left(\Omega,\left\{\mathscr{F}_{t}\right\}_{t>0}, P\right)$ be a complete probability space with a filtration $\left\{\mathscr{F}_{t}\right\}_{t \geq 0}$ satisfying the usual conditions (i.e., it is right continuous and $\mathscr{F}_{0}$ contains all $P$-null sets). Let $R_{+}^{2}$ denote the positive cone of $R^{2}$; namely, $R_{+}^{2}=\left\{x=\left(x_{1}, x_{2}\right) \in R^{2}\right.$ : $\left.x_{i}>0, i=1,2\right\}, \bar{R}_{+}^{2}=\left\{x=\left(x_{1}, x_{2}\right) \in R^{2}: x_{i} \geq 0, i=1,2\right\}$.

\section{Existence and Uniqueness of the Nonnegative Solution}

To investigate the dynamical behavior, first, we should concern whether the solution is global existence. Moreover, for a population model, we should also consider whether the solution is nonnegative. Hence, in this section we show that the solution of system (3) is global and nonnegative. As we have known, in order for a stochastic differential equation to have a unique global (i.e., no explosion at a finite time) solution with any given initial value, the coefficients of the equation are generally required to satisfy the linear growth condition and local Lipschitz condition (see, e.g., [17]). It is easy to see that the coefficients of system (3) are locally Lipschitz continuous, so system (3) has a local solution. By Lyapunov analysis method, we show the global existence of this solution.

By the classical comparison theorem of stochastic differential equations, we could get the following.

Lemma 1. Let $(x(t), y(t))$ be a positive solution of system (3) with $(x(0), y(0)) \in R_{+}^{2}$. Then, we have

$$
\begin{gathered}
x(t) \leq X(t), \\
y(t) \leq Y(t), \quad \text { a.s., }
\end{gathered}
$$

where $(X(t), Y(t))$ are solutions of the following stochastic differential equations:

$$
\begin{array}{r}
\dot{X}(t)=r X(t)\left(1-\frac{X(t)}{K}\right)+\frac{\sigma_{1}}{K} X(t)(K-X(t)) \dot{B}_{1}(t), \\
X(0)=x(0), \\
\dot{Y}(t)=-\mu Y(t)+m c X(t) Y(t)-\sigma_{2} Y(t) \dot{B}_{2}(t), \\
Y(0)=y(0) .
\end{array}
$$

Consider the stochastic logistic equation

$$
\begin{array}{r}
d N(t)=N(t)\left[\left(r-\frac{r}{K} N(t)\right) d t+\frac{\alpha}{K}(K-N(t)) d B(t)\right], \\
r, K>0 .
\end{array}
$$

Jiang et al. [18] studied system (6) and obtained the following result.

Lemma 2. There exists a unique continuous positive solution $0<N(t)<K$ to system (6) for any initial value $N(0)=N_{0}$ with $0<N_{0}<K$. If $r>\alpha^{2} / 2$, then

$$
\lim _{t \rightarrow \infty} N(t)=K, \quad \text { a.s. }
$$

From Lemmas 1 and 2, it is easy to get the following result.

Lemma 3. Let $(x(t), y(t))$ be a positive solution of system (3) with $0<x(0)<K$. Then, we have

$$
0<x(t)<K \text {. a.s. }
$$

Theorem 4. For any initial value $\left\{(x(0), y(0)) \in R_{+}^{2}, x(0) \in\right.$ $(0, K)\}$, there is a unique solution $(x(t), y(t))$ of system (3) on $t \geq 0$, and the solution will remain in $R_{+}^{2}$ with probability 1 . 
Proof. It is clear that the coefficients of system (3) are locally Lipschitz continuous for the given initial value $\{(x(0), y(0)) \in$ $\left.R_{+}^{2}, x(0) \in(0, K)\right\}$. So there is a unique local solution $(x(t), y(t))$ on $t \in\left[0, \tau_{e}\right)$, where $\tau_{e}$ is the explosion time (see, e.g., [17]). To show this solution is global, we need to show that $\tau_{e}=\infty$ a.s. Let $k_{0} \geq 1$ be sufficiently large so that $x(0)$ and $y(0)$ all lie within the interval $\left[1 / k_{0}, k_{0}\right]$. For each integer $k \geq k_{0}$, define the stopping time

$$
\begin{aligned}
\tau_{m}=\inf \left\{t \in\left[0, \tau_{e}\right)\right. & : \min \{x(t), y(t)\} \\
\leq & \left.\frac{1}{k} \text { or } \max \{x(t), y(t)\} \geq k\right\} .
\end{aligned}
$$

Throughout this paper, we set inf $\emptyset=\infty$ (as usual $\emptyset$ denotes the empty set). Clearly, $\tau_{k}$ is increasing as $k \rightarrow \infty$. Set $\tau_{\infty}=$ $\lim _{k \rightarrow \infty} \tau_{k}$; then, $\tau_{\infty} \leq \tau_{e}$ a.s. If we can show that $\tau_{\infty}=\infty$ a.s., then $\tau_{e}=\infty$ and $(x(t), y(t)) \in R_{+}^{2}$ a.s. for all $t \geq 0$. In other words, to complete the proof all we need to show is that $\tau_{\infty}=\infty$ a.s. If this statement is false, then there is a pair of constants $T>0$ and $\epsilon \in(0,1)$ such that

$$
P\left\{\tau_{\infty} \leq T\right\}>\epsilon .
$$

Hence, there is an integer $k_{1} \geq k_{0}$ such that

$$
P\left\{\tau_{k} \leq T\right\} \geq \epsilon \quad \forall k \geq k_{1} .
$$

Define a $C^{2}$-function $V: R_{+}^{2} \rightarrow \bar{R}_{+}$by

$$
V(x, y)=\left(x-a-a \log \frac{x}{a}\right)+\frac{1}{m}(y-1-\log y),
$$

where $a$ is a positive constant to be determined later. The nonnegativity of this function can be seen from $u-1-\log u \geq$ 0 , for all $u>0$. Using Itô's formula, we get

$$
\begin{aligned}
d V:= & L V d t+\frac{\sigma_{1}}{K}(x-a)(K-x) d B_{1}(t) \\
& +\frac{\sigma_{2}}{m}(y-1) d B_{2}(t),
\end{aligned}
$$

where

$$
\begin{aligned}
L V= & (x-a)\left(r-\frac{r}{K} x-c y\right)+\frac{a \sigma_{1}^{2}}{2 K^{2}}(K-x)^{2} \\
& +\frac{1}{m}(y-1)(-\mu+m c x)+\frac{\sigma_{2}^{2}}{2 m} \\
= & -a r+\frac{a \sigma_{1}^{2}}{2}+\frac{\mu}{m}+\frac{\sigma_{2}^{2}}{2 m}+\left(r+\frac{a r-a \sigma_{1}^{2}}{K}-c\right) x \\
& -\left(\frac{r}{K}-\frac{a \sigma_{1}^{2}}{2 K^{2}}\right) x^{2}-\left(\frac{\mu}{m}-a c\right) y .
\end{aligned}
$$

Choose $a=\mu / m c$ such that $\mu / m-a c=0$, together with Lemma 3; then,

$$
\begin{aligned}
L V \leq & -a r+\frac{a \sigma_{1}^{2}}{2}+\frac{\mu}{m}+\frac{\sigma_{2}^{2}}{2 m} \\
& +\left(r+\frac{a r-a \sigma_{1}^{2}}{K}-c\right) x-\left(\frac{r}{K}-\frac{a \sigma_{1}^{2}}{2 K^{2}}\right) x^{2} \leq M,
\end{aligned}
$$

where $M$ is a positive constant. Therefore,

$$
\begin{aligned}
& \int_{0}^{\tau_{k} \wedge T} d V(x(t), y(t)) \\
& \leq \int_{0}^{\tau_{k} \wedge T} M d t+\int_{0}^{\tau_{k} \wedge T} \frac{\sigma_{1}}{K}\left(x-\frac{\mu}{m c}\right)(K-x) d B_{1}(s) \\
& \quad+\frac{\sigma_{2}}{m}(y(s)-1) d B_{2}(s), \\
& E\left[V\left(x\left(\tau_{k} \wedge T\right), y\left(\tau_{k} \wedge T\right)\right)\right] \\
& \leq V(x(0), y(0)) \\
& \quad+E \int_{0}^{\tau_{k} \wedge T} K d t \leq V(x(0), y(0))+M T .
\end{aligned}
$$

Set $\Omega_{k}=\left\{\tau_{k} \leq T\right\}$ for $k \geq k_{1}$; then, by (11), we know that $P\left(\Omega_{k}\right) \geq \epsilon$. Note that for every $\omega \in \Omega_{k}$, there is at least one of $x\left(\tau_{k}, \omega\right)$ and $y\left(\tau_{k}, \omega\right)$ equals either $k$ or $1 / k$; then,

$$
\begin{aligned}
V\left(x\left(\tau_{k}\right), y\left(\tau_{k}\right)\right) & \geq\left(k-a-a \log \frac{k}{a}\right) \\
& \wedge\left(\frac{1}{k}-a+a \log (a k)\right) \\
& \wedge \frac{1}{k}(k-1-\log k) \\
& \wedge \frac{1}{k}\left(\frac{1}{k}-1+\log k\right) .
\end{aligned}
$$

It then follows from (11) and (16) that

$$
\begin{aligned}
V(x(0), y(0))+K T & \geq E\left[1_{\Omega_{k}(\omega)} V\left(x\left(\tau_{k}\right), y\left(\tau_{k}\right)\right)\right] \\
& \geq \epsilon\left(k-a-a \log \frac{k}{a}\right) \\
& \wedge\left(\frac{1}{k}-a+a \log (a k)\right) \\
& \wedge \frac{1}{k}(k-1-\log k) \\
& \wedge \frac{1}{k}\left(\frac{1}{k}-1+\log k\right),
\end{aligned}
$$

where $1_{\Omega_{k}(\omega)}$ is the indicator function of $\Omega_{k}$. Letting $k \rightarrow \infty$ leads to the contradiction that $\infty>V(x(0), y(0))+M T=\infty$. So we must, therefore, have $\tau_{\infty}=\infty$ a.s.

\section{Permanence}

There is no equilibrium of system (3). Hence, we cannot show the permanence of the system by proving the stability of the positive equilibrium as the deterministic system. In this section we first show that this system is persistent in mean.

3.1. Persistent in Time Average. L. S. Chen and J. Chen in [19] proposed the definition of persistence in mean for the 
deterministic system. Here, we also use this definition for the stochastic system.

Definition 5. System (3) is said to be persistent in mean if

$$
\liminf _{t \rightarrow \infty} \frac{1}{t} \int_{0}^{t} y(s) d s>0, \quad \text { a.s. }
$$

Lemma 6 (Xia et al. [20, Lemma 17]). Let $f \in C([0,+\infty) \times$ $\Omega,(0,+\infty)), F \in C([0,+\infty) \times \Omega, R)$. If there exist positive constants $\lambda_{0}, \lambda$, such that

$$
\log f(t) \geq \lambda t-\lambda_{0} \int_{0}^{t} f(s) d s+F(t), \quad t \geq 0 \text { a.s. }
$$

and $\lim _{t \rightarrow \infty}(F(t) / t)=0$ a.s., then

$$
\liminf _{t \rightarrow \infty} \frac{1}{t} \int_{0}^{t} f(s) d s \geq \frac{\lambda}{\lambda_{0}}, \quad \text { a.s. }
$$

Assumption 7. We have

$$
\left(r-\frac{\sigma_{1}^{2}}{2}\right) K m c-r\left(\mu+\frac{\sigma_{2}^{2}}{2}\right)>0 .
$$

Theorem 8. If Assumption 7 is satisfied, then the solution $(x(t), y(t))$ of system (3) with any initial value $\{(x(0), y(0)) \in$ $\left.R_{+}^{2}, x(0) \in(0, K)\right\}$ has the following property:

$$
\begin{aligned}
\liminf _{t \rightarrow \infty} \frac{1}{t} \int_{0}^{t} y(s) d s & \geq \frac{\left(r-\left(\sigma_{1}^{2} / 2\right)\right) K m c-r\left(\mu+\left(\sigma_{1}^{2} / 2\right)\right)}{K m c^{2}} \\
& >0, \quad \text { a.s. }
\end{aligned}
$$

Proof. According to Ito's formula, the system (3) is changed into

$$
\begin{gathered}
d \log x(t)=r-\frac{r}{K} x(t)-c y(t) \\
\quad-\frac{\sigma_{1}^{2}}{2 K^{2}}(K-x(t))^{2}+\frac{\sigma_{1}}{K}(K-x(t)) d B_{1}(t), \\
d \log y(t)=-\mu-\frac{\sigma_{2}^{2}}{2}+m c x(t)-\sigma_{2} d B_{2}(t)
\end{gathered}
$$

then,

$$
\begin{aligned}
d( & \left.\log x(t)+\frac{r}{K m c} \log y(t)\right) \\
= & r-\frac{r}{K m c}\left(\mu+\frac{\sigma_{2}^{2}}{2}\right)-c y(t)-\frac{\sigma_{1}^{2}}{2 K^{2}}(K-x(t))^{2} \\
& +\frac{\sigma_{1}}{K}(K-x(t)) d B_{1}(t)-\frac{r \sigma_{2}}{K m c} d B_{2}(t) \\
\geq & \left(r-\frac{r}{K m c}\left(\mu+\frac{\sigma_{2}^{2}}{2}\right)-\frac{\sigma_{1}^{2}}{2}\right) \\
& -c y(t)+\frac{\sigma_{1}}{K}(K-x(t)) d B_{1}(t)-\frac{r \sigma_{2}}{K m c} d B_{2}(t) .
\end{aligned}
$$

After that

$$
\begin{aligned}
& \frac{\log x(t)-\log x(0)}{t}+\frac{r}{K m c} \frac{\log y(t)-\log y(0)}{t} \\
& \geq\left(r-\frac{r}{K m c}\left(\mu+\frac{\sigma_{2}^{2}}{2}\right)-\frac{\sigma_{1}^{2}}{2}\right)-c \frac{\int_{0}^{t} y(s) d s}{t} \\
& \quad+\frac{\sigma_{1}}{K t} \int_{0}^{t}(K-x(s)) d B_{1}(s)-\frac{r \sigma_{2}}{K m c t} \int_{0}^{t} d B_{2}(s) ;
\end{aligned}
$$

besides, from Lemma 3, it is clear that

$$
\limsup _{t \rightarrow \infty} \frac{\log x(t)}{t} \leq 0,
$$

where $M_{1}(t)=\int_{0}^{t}(K-x(s)) d B_{1}(s)$ and $M_{2}(t)=\int_{0}^{t} d B_{2}(s)$ are martingale with $M_{i}(0)=0(i=1,2)$, and from Lemma 3 we get

$$
\limsup _{t \rightarrow \infty} \frac{\left\langle M_{1}, M_{1}\right\rangle}{t},=\limsup _{t \rightarrow \infty} \frac{1}{t} \int_{0}^{t}(K-x(s))^{2} d s \leq K^{2} ;
$$

then, by strong law of large numbers, we know that $\lim _{t \rightarrow \infty}\left(M_{i} / t\right)=0(i=1,2)$.

Hence,

$$
\begin{aligned}
\lim _{t \rightarrow 0} & \left(\left(r-(r / K m c)\left(\mu+\left(\sigma_{2}^{2} / 2\right)\right)-\left(\sigma_{1}^{2} / 2\right)\right) t\right. \\
& \left.+\left(\sigma_{1} / K\right) M_{1}(t)-\left(r \sigma_{2} / K m c\right) M_{2}(t)\right) \times(t)^{-1} \\
= & r-\frac{r}{K m c}\left(\mu+\frac{\sigma_{2}^{2}}{2}\right)-\frac{\sigma_{1}^{2}}{2} .
\end{aligned}
$$

With Lemma 6 and Assumption 7 we could get

$$
\begin{aligned}
\lim _{t \rightarrow \infty} & \frac{1}{t} \int_{0}^{t} y(s) d s \\
\geq & \frac{r-\left(\sigma_{1}^{2} / 2\right)-(r / K m c)\left(\mu+\left(\sigma_{2}^{2} / 2\right)\right)}{c} \\
& =\frac{\left(r-\left(\sigma_{1}^{2} / 2\right)\right) K m c-r\left(\mu+\left(\sigma_{2}^{2} / 2\right)\right)}{K m c^{2}}>0 .
\end{aligned}
$$

3.2. Stationary Distribution and Ergodicity for System (3). In this section we show there is a stationary distribution of system (3).

Theorem 9. Let $(x(t), y(t))$ be the solution of system (3) with any initial value $\left\{(x(0), y(0)) \in R_{+}^{2}, x(0) \in(0, K)\right\}$. If $\sigma_{2}^{2}<$ $\mu<\min \left\{m c K, r m c K / \sigma_{1}^{2}\right\}$ and $\sigma_{1}>0, \sigma_{2}>0$, such that $\sigma_{1}^{2}<$ $\mathrm{Kr} / \mathrm{x}^{*}$ and

$$
\begin{aligned}
\left(\frac{1}{2}+l x^{*}\right) x^{*} \sigma_{1}^{2}+\left(\frac{1}{2}+\frac{l y^{*}}{m}\right) \frac{y^{*} \sigma_{2}^{2}}{m} \\
<\min \left\{\frac{1}{2}\left(\frac{r}{K}-\frac{x^{*} \sigma_{1}^{2}}{K^{2}}\right)\left(x^{*}\right)^{2},\right. \\
\\
\left.\frac{l}{2}\left(\mu-\sigma_{2}^{2}\right)\left(y^{*}\right)^{2},\left(K-x^{*}\right)^{2}\right\},
\end{aligned}
$$


where $\left(x^{*}, y^{*}\right)$ is the positive equilibrium of system (1) and $l$ is defined as in the proof, then system (3) exists as a stationary distribution and it is ergodic.

Proof. Since $\mu<m c K$, then there is a positive equilibrium $\left(x^{*}, y^{*}\right)$ of system (1), and

$$
r=\frac{r}{K} x^{*}+c y^{*}, \quad \mu=m c x^{*} .
$$

Define

$$
\begin{aligned}
V_{1}(x, y)= & \left(x-x^{*}-x^{*} \log \frac{x}{x^{*}}\right) \\
& +\frac{1}{m}\left(y-y^{*}-y^{*} \log \frac{y}{y^{*}}\right),
\end{aligned}
$$

and let $L$ be the generating operator of system (3). Then,

$$
\begin{aligned}
L V_{1}= & \left(x-x^{*}\right)\left(r-\frac{r}{K} x-c y\right) \\
& +\frac{x^{*} \sigma_{1}^{2}}{2 K^{2}}(K-x)^{2}+\frac{1}{m}\left(y-y^{*}\right)(-\mu+m c x)+\frac{y^{*} \sigma_{2}^{2}}{2 m} \\
= & \left(x-x^{*}\right)\left[-\frac{r}{K}\left(x-x^{*}\right)-c\left(y-y^{*}\right)\right] \\
& +\frac{x^{*} \sigma_{1}^{2}}{2 K^{2}}\left(K-x^{*}-\left(x-x^{*}\right)\right)^{2} \\
& +c\left(x-x^{*}\right)\left(y-y^{*}\right)+\frac{y^{*} \sigma_{2}^{2}}{2 m} \\
\leq & -\frac{r}{K}\left(x-x^{*}\right)^{2}+\frac{x^{*} \sigma_{1}^{2}}{K^{2}} \\
& \times\left(\left(K-x^{*}\right)^{2}+\left(x-x^{*}\right)^{2}\right)+\frac{y^{*} \sigma_{2}^{2}}{2 m} \\
= & -\left(\frac{r}{K}-\frac{x^{*} \sigma_{1}^{2}}{K^{2}}\right)\left(x-x^{*}\right)^{2} \\
& +\frac{x^{*} \sigma_{1}^{2}}{2 K^{2}}\left(K-x^{*}\right)^{2}+\frac{y^{*} \sigma_{2}^{2}}{2 m} .
\end{aligned}
$$

Define

$$
V_{2}(x, y)=\frac{1}{2}\left[\left(x-x^{*}\right)+\frac{1}{k}\left(y-y^{*}\right)\right]^{2} ;
$$

Note that

$$
\begin{aligned}
& d\left[\left(x-x^{*}\right)+\frac{1}{m}\left(y-y^{*}\right)\right] \\
& =\left(r x-\frac{r}{K} x^{2}-\frac{\mu}{m} y\right) d t \\
& \quad+\frac{\sigma_{1}}{K} x(K-x) d B_{1}(t)-\frac{\sigma_{2}}{m} y d B_{2}(t) \\
& =\left[r\left(x-x^{*}\right)-\frac{r}{K}\left(x^{2}-\left(x^{*}\right)^{2}\right)-\frac{\mu}{m}\left(y-y^{*}\right)\right] d t \\
& \quad+\frac{\sigma_{1}}{K} x(K-x) d B_{1}(t)-\frac{\sigma_{2}}{m} y d B_{2}(t)
\end{aligned}
$$

$$
\begin{aligned}
= & {\left[r\left(x-x^{*}\right)-\frac{r}{K}\left(\left(x-x^{*}\right)^{2}+2 x^{*}\left(x-x^{*}\right)\right)\right.} \\
& \left.-\frac{\mu}{m}\left(y-y^{*}\right) d t\right] \\
& +\frac{\sigma_{1}}{K} x(K-x) d B_{1}(t)-\frac{\sigma_{2}}{m} y d B_{2}(t) \\
= & {\left[\left(r-\frac{2 r x^{*}}{K}\right)\left(x-x^{*}\right)-\frac{r}{K}\left(x-x^{*}\right)^{2}-\frac{\mu}{m}\left(y-y^{*}\right)\right] d t } \\
& +\frac{\sigma_{1}}{K} x(K-x) d B_{1}(t)-\frac{\sigma_{2}}{m} y d B_{2}(t) ;
\end{aligned}
$$

Then,

$$
\begin{aligned}
L V_{2}= & {\left[\left(x-x^{*}\right)+\frac{1}{m}\left(y-y^{*}\right)\right] } \\
& \times\left[\left(r-\frac{2 r x^{*}}{K}\right)\left(x-x^{*}\right)\right. \\
& \left.\quad-\frac{r}{K}\left(x-x^{*}\right)^{2}-\frac{\mu}{m}\left(y-y^{*}\right)\right] \\
& +\frac{\sigma_{1}^{2}}{2 K^{2}} x^{2}(K-x)^{2}+\frac{\sigma_{2}^{2}}{2 m^{2}} y^{2} \\
\leq & \left(r-\frac{2 r x^{*}}{K}\right)\left(x-x^{*}\right)^{2}+\left(\frac{r}{m}-\frac{2 r}{K m} x^{*}-\frac{\mu}{m}\right) \\
& \times\left(x-x^{*}\right)\left(y-y^{*}\right)-\frac{\mu}{m^{2}}\left(y-y^{*}\right)^{2} \\
& +\frac{\sigma_{1}^{2}}{2 K^{2}} x^{2}(K-x)^{2}+\frac{\sigma_{2}^{2}}{2 m^{2}} y^{2} \\
\leq & r\left(x-x^{*}\right)^{2}+\left(\frac{r}{m}-\frac{2 r}{K m} x^{*}-\frac{\mu}{m}\right) \\
& \times\left(x-x^{*}\right)\left(y-y^{*}\right)-\frac{\mu}{m^{2}}\left(y-y^{*}\right)^{2} \\
& +\sigma_{1}^{2}\left[\left(x-x^{*}\right)^{2}+\left(x^{*}\right)^{2}\right]+\frac{\sigma_{2}^{2}}{m^{2}}\left[\left(y-y^{*}\right)^{2}+\left(y^{*}\right)^{2}\right] \\
= & \left(r+\sigma_{1}^{2}\right)\left(x-x^{*}\right)^{2}-\frac{\mu-\sigma_{2}^{2}}{m^{2}}\left(y-y^{*}\right)^{2} \\
& +\left(\frac{r}{m}-\frac{2 r}{K m} x^{*}-\frac{\mu}{m}\right)\left(x-x^{*}\right)\left(y-y^{*}\right) \\
& +\sigma_{1}^{2}\left(x^{*}\right)^{2}+\frac{\sigma_{2}^{2}}{m^{2}}\left(y^{*}\right)^{2},
\end{aligned}
$$

where $L$ is also the generating operator of system (3). Note that

$$
\begin{aligned}
& \left(\frac{r}{m}-\frac{2 r}{K m} x^{*}-\frac{\mu}{m}\right)\left(x-x^{*}\right)\left(y-y^{*}\right) \\
& \quad \leq \frac{\left(r / m-(2 r / K m) x^{*}-\mu / m\right)^{2}}{2\left(\left(\mu-\sigma_{2}^{2}\right) / m^{2}\right)}\left(x-x^{*}\right)^{2}
\end{aligned}
$$




$$
\begin{aligned}
& +\frac{1}{2}\left(\frac{\mu-\sigma_{2}^{2}}{m^{2}}\right)\left(y-y^{*}\right)^{2} \\
:= & \delta\left(x-x^{*}\right)^{2}+\left(\frac{\mu-\sigma_{2}^{2}}{2 m^{2}}\right)\left(y-y^{*}\right)^{2} ;
\end{aligned}
$$

Then,

$$
\begin{aligned}
L V_{2} \leq & \left(r+\sigma_{1}^{2}+\delta\right)\left(x-x^{*}\right)^{2} \\
& -\left(\frac{\mu-\sigma_{2}^{2}}{2 m^{2}}\right)\left(y-y^{*}\right)^{2}+\sigma_{1}^{2}\left(x^{*}\right)^{2}+\frac{\sigma_{2}^{2}}{m^{2}}\left(y^{*}\right)^{2}
\end{aligned}
$$

Now define

$$
V(x, y)=V_{1}(x, y)+l V_{2}(x, y)
$$

where $l$ is a positive constant to be determined later. Then,

$$
\begin{aligned}
L V \leq & -\left(\frac{r}{K}-\frac{x^{*} \sigma_{1}^{2}}{K^{2}}-l\left(r+\sigma_{1}^{2}+\delta\right)\right)\left(x-x^{*}\right)^{2} \\
& -\frac{l}{2}\left(\frac{\mu-\sigma_{2}^{2}}{m^{2}}\right)\left(y-y^{*}\right)^{2}+\left(\frac{1}{2}+l x^{*}\right) x^{*} \sigma_{1}^{2} \\
& +\left(\frac{1}{2}+\frac{l y^{*}}{m}\right) \frac{y^{*} \sigma_{2}^{2}}{m} .
\end{aligned}
$$

Choose $l>0$ such that $\left((r / K)-\left(x^{*} \sigma_{1}^{2} / K^{2}\right)-l\left(r+\sigma_{1}^{2}+\delta\right)\right)=$ $(1 / 2)\left((r / K)-\left(x^{*} \sigma_{1}^{2} / K^{2}\right)\right)$. Then, it follows from (47) that

$$
\begin{aligned}
L V \leq & -\frac{1}{2}\left(\frac{r}{K}-\frac{x^{*} \sigma_{1}^{2}}{K^{2}}\right)\left(x-x^{*}\right)^{2} \\
& -\frac{l}{2}\left(\frac{\mu-\sigma_{2}^{2}}{m^{2}}\right)\left(y-y^{*}\right)^{2}+\left(\frac{1}{2}+l x^{*}\right) x^{*} \sigma_{1}^{2} \\
& +\left(\frac{1}{2}+\frac{l y^{*}}{m}\right) \frac{y^{*} \sigma_{2}^{2}}{m} .
\end{aligned}
$$

Note that

$$
\begin{aligned}
\left(\frac{1}{2}+l x^{*}\right) x^{*} \sigma_{1}^{2}+\left(\frac{1}{2}+\frac{l y^{*}}{m}\right) \frac{y^{*} \sigma_{2}^{2}}{m} \\
<\min \left\{\frac{1}{2}\left(\frac{r}{K}-\frac{x^{*} \sigma_{1}^{2}}{K^{2}}\right)\left(x^{*}\right)^{2},\right. \\
\left.\frac{l}{2}\left(\frac{\mu-\sigma_{2}^{2}}{m^{2}}\right)\left(y^{*}\right)^{2},\left(K-x^{*}\right)^{2}\right\} .
\end{aligned}
$$

Then, the ellipsoid

$$
\begin{aligned}
& -\frac{1}{2}\left(\frac{r}{K}-\frac{x^{*} \sigma_{1}^{2}}{K^{2}}\right)\left(x-x^{*}\right)^{2}-\frac{l}{2}\left(\frac{\mu-\sigma_{2}^{2}}{m^{2}}\right)\left(y-y^{*}\right)^{2} \\
& +\left(\frac{1}{2}+l x^{*}\right) x^{*} \sigma_{1}^{2}+\left(\frac{1}{2}+\frac{l y^{*}}{m}\right) \frac{y^{*} \sigma_{2}^{2}}{m}=0
\end{aligned}
$$

lies entirely in $D^{0}=\left\{(x, y) \in R_{+}^{2} \mid 0<x<K\right\}$. We can take $U$ to be a neighborhood of the ellipsoid with $\bar{U} \subseteq E_{l}=D^{0}$, so that for $x \in U \backslash E_{l}, L V \leq-C$ (C is a positive constant), which implies that condition (B.2) in Lemma 3.2 of [21] is satisfied. Hence, the solution $(x(t), y(t))$ is recurrent in the domain $U$, which together with Lemma 3.3 and Remark 3.3 of [21] imply that $(x(t), y(t))$ is recurrent in any bounded domain $D \subset D^{0}$. Besides, for $\forall D$, there is a $M=\min \left\{\left(x^{2} \sigma_{1}^{2} / K^{2}\right)(K-x)^{2}, \sigma_{2}^{2} y^{2},(x, y) \in \bar{D}\right\}>0$, such that

$$
\begin{aligned}
\sum_{i, j=1}^{2} \lambda_{i j} \xi_{i} \xi_{j} & =\frac{x^{2} \sigma_{1}^{2}}{K^{2}}(K-x)^{2} \xi_{1}^{2}+\sigma_{2}^{2} y^{2} \xi_{2}^{2} \\
& \geq M\left|\xi^{2}\right| \quad \forall x \in \bar{D}, \quad \xi \in R^{2},
\end{aligned}
$$

which implies that condition (B.1) in Lemma 3.2 of [21] is also satisfied. Therefore, system (3) has a stationary distribution $\mu(\cdot)$ and it is ergodic.

From Lemma 3, with the initial value $0<x(0)<K$, we have the property

$$
0<x(t)<K \quad \text { a.s. }
$$

Therefore, by ergodicity property, we know that function $f(z)=z^{p}$ is integrable with respect to the measure $\mu$, and

$$
\lim _{t \rightarrow \infty} \frac{1}{t} \int_{0}^{t} x^{p}(s) d s=\int_{R_{+}^{2}} z^{p} \mu\left(d z_{1}, d z_{2}\right), \quad \text { a.s. }
$$

Hence, from these arguments, we get the following result.

Theorem 10. Assume the same conditions as in Theorem 9. Then, we have

$$
\lim _{t \rightarrow \infty} \frac{1}{t} \int_{0}^{t} x^{p}(s) d s=\int_{R_{+}^{2}} z^{p} \mu\left(d z_{1}, d z_{2}\right), \quad \text { a.s. }
$$

\section{Extinction}

In this section, we show the situation when the population of system (3) will be extinct. Before we give the result, we should do some prepare work. We first introduce a result on the Feller's test (see, e.g., [22]).

Let $I=(l, r),-\infty \leq r \leq+\infty$. Consider the following one-dimensional time-homogeneous stochastic differential equation:

$$
d X_{t}=\mu\left(X_{t}\right) d t+\sigma\left(X_{t}\right) d B_{t}, \quad X_{0}=x
$$

Assume that the coefficients $\sigma: I \rightarrow R, \mu: I \rightarrow R$ satisfy the following conditions:

$$
\text { (1) } \sigma^{2}(x)>0 ; \quad \forall x \in I,
$$

(2) $\forall x \in I, \exists \varepsilon>0, \quad \int_{x-\varepsilon}^{x+\varepsilon} \frac{1+\mu(y)}{\sigma^{2}(y)} d s<\infty$. 
Fixing some $c \in I$, the scale function is defined by

$$
p(x)=\int_{c}^{x} e^{-\int_{c}^{v}\left(2 \mu(y) / \sigma^{2}(y)\right) d y} d v, \quad x \in R .
$$

Now, we present a useful Lemma.

Lemma 11. Assume that conditions (1) and (2) hold in (50), and let $X$ be a nonexplosive solution of system (49) in I, with $X_{0}=x \in I ;$ we distinguish four cases:

(a) $p(l+)=-\infty, p(r-)=+\infty$, then $P\left\{\sup _{t \geq 0} X_{t}=r\right\}=$ $P\left\{\inf _{t \geq 0} X_{t}=l\right\}=1$, and for any $y \in I$, we have $p\left\{\exists t \in(0, \infty), X_{t}=y\right\}=1$.

(b) $p(l+)>-\infty, p(r-)=+\infty$, then $P\left\{\sup _{t \geq 0} X_{t}=l\right\}=$ $P\left\{\inf _{t \geq 0} X_{t}<r\right\}=1$.

(c) $p(l+)=-\infty, p(r-)<+\infty$, then $P\left\{\sup _{t \geq 0} X_{t}=r\right\}=$ $P\left\{\inf _{t \geq 0} X_{t}>l\right\}=1$.

(d) $p(l+)>-\infty, p(r-)<+\infty$, then $P\left\{\sup _{t \geq 0} X_{t}=l\right\}=$ $1-P\left\{\inf _{t \geq 0} X_{t}=r\right\}=(p(r-)-p(x)) /(p(r-)-p(l+))$.

Case $1\left(r<\sigma_{1}^{2} / 2\right)$. Consider the first equation of system (5). Let

$$
X(t)=\frac{K e^{Z(t)}}{1+e^{Z(t)}}
$$

Then,

$$
Z(t)=\log \frac{X(t)}{K-X(t)},
$$

and so the first equation of system (5) is reformed as

$$
d Z(t)=\left(r-\frac{\sigma_{1}^{2}}{2}+\frac{e^{Z(t)}}{1+e^{Z(t)}} \sigma_{1}^{2}\right) d t+\sigma_{1} d B_{1}(t), \quad t \geq 0
$$

with an initial value $Z(0)=\log (x(0) /(K-x(0)))$. Let

$$
\mu(x)=r-\frac{\sigma_{1}^{2}}{2}+\frac{e^{x}}{1+e^{x}} \sigma_{1}^{2}, \quad \sigma(x)=\sigma_{1} .
$$

Then,

$$
\begin{aligned}
& \int_{0}^{v} \frac{-2 \mu(x)}{\sigma^{2}(x)} d x \\
& \quad=\frac{-2}{\sigma_{1}^{2}} \int_{0}^{v}\left(r-\frac{\sigma_{1}^{2}}{2}+\frac{e^{x}}{1+e^{x}} \sigma_{1}^{2}\right) d x \\
& \quad=\frac{-2}{\sigma_{1}^{2}}\left[\left(r-\frac{\sigma_{1}^{2}}{2}\right) v+\sigma_{1}^{2} \log \left(1+e^{v}\right)\right]+2 \log 2 .
\end{aligned}
$$

So if $r_{1}<\sigma_{1}^{2} / 2$, by Lemma 11 , we get $p(-\infty)>-\infty$, $p(+\infty)<$ $+\infty$; then,

$$
\begin{aligned}
P\left\{\sup _{t \geq 0} Z(t)=-\infty\right\} & =1-P\left\{\inf _{t \geq 0} Z(t)=+\infty\right\} \\
& =\frac{p(\infty)-p(x)}{p(\infty)-p(-\infty)} .
\end{aligned}
$$

Hence,

$$
\begin{aligned}
P\left\{\lim _{t \rightarrow \infty} X(t)=0\right\} & =1-P\left\{\lim _{t \rightarrow \infty} X(t)=K\right\} \\
& =\frac{p(\infty)-p(x)}{p(\infty)-p(-\infty)} \\
& =\frac{\int_{Z(0)}^{+\infty} e^{\left(-2 / \sigma_{1}^{2}\right)\left(r-\sigma_{1}^{2} / 2\right) v}\left(1+e^{v}\right)^{-2} d v}{\int_{-\infty}^{+\infty} e^{\left(-2 / \sigma_{1}^{2}\right)\left(r-\sigma_{1}^{2} / 2\right) v}\left(1+e^{v}\right)^{-2} d v} .
\end{aligned}
$$

Furthermore, by the classical comparison theorem of stochastic differential equations, we have

$$
x(t) \leq X(t), \quad y(t) \leq Y(t),
$$

where $(x(t), y(t))$ is the solution of system (3). We could get

$$
P\left\{\lim _{t \rightarrow \infty} x(t)=0\right\} \geq \frac{\int_{Z(0)}^{+\infty} e^{\left(-2 / \sigma_{1}^{2}\right)\left(r-\sigma_{1}^{2} / 2\right) v}\left(1+e^{v}\right)^{-2} d v}{\int_{-\infty}^{+\infty} e^{\left(-2 / \sigma_{1}^{2}\right)\left(r-\sigma_{1}^{2} / 2\right) v}\left(1+e^{v}\right)^{-2} d v} .
$$

So, if $\lim _{k \rightarrow \infty} x(t)=0$, a.s., $\omega \in \Omega_{0}=\left\{\omega: \lim _{k \rightarrow \infty} x(t)=0\right\}$, and from (24), then we know

$$
d \log y(t)=-\mu-\frac{\sigma_{2}^{2}}{2}+m c x(t)-\sigma_{2} d B_{2}(t) .
$$

Then,

$$
\limsup _{t \rightarrow \infty} \frac{\log y(t)}{t}=-\mu-\frac{\sigma_{2}^{2}}{2}<0, \quad \omega \in \Omega_{0} .
$$

Therefore, with the condition $r-\sigma_{1}^{2} / 2<0$, we obtain the fact that system (3) will be extinct in probability.

Case $2\left(r>\sigma_{1}^{2} / 2, \mu+\sigma_{2}^{2} / 2>m c K\right)$. According to Ito's formula and comparison principle, the second population of system (3) is changed into

$$
\begin{aligned}
d \log y(t) & =-\mu-\frac{\sigma_{2}^{2}}{2}+m c x(t)-\sigma_{2} d B_{2}(t) \\
& \leq-\mu-\frac{\sigma_{2}^{2}}{2}+m c X(t)-\sigma_{2} d B_{2}(t) .
\end{aligned}
$$

Notice that $X(t)<K$ and then let (63) be divided by $t, t \rightarrow$ $\infty$; we could get

$$
\limsup _{t \rightarrow \infty} \frac{\log y(t)}{t} \leq-\mu-\frac{\sigma_{2}^{2}}{2}+m c K \quad \text { a.s. }
$$

If $\mu+\sigma_{2}^{2} / 2>m c K$, it follows

$$
\limsup _{t \rightarrow \infty} \frac{\log y(t)}{t}<0 \quad \text { a.s.; }
$$

hence,

$$
\lim _{t \rightarrow \infty} y(t)=0, \quad \text { a.s. }
$$




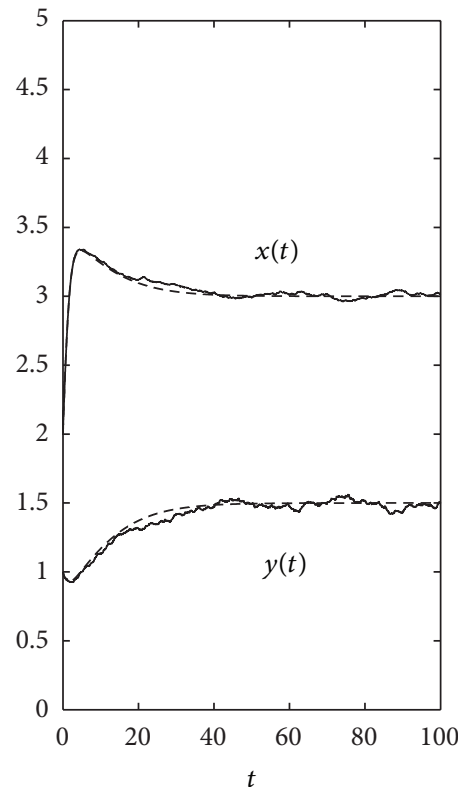

(a)

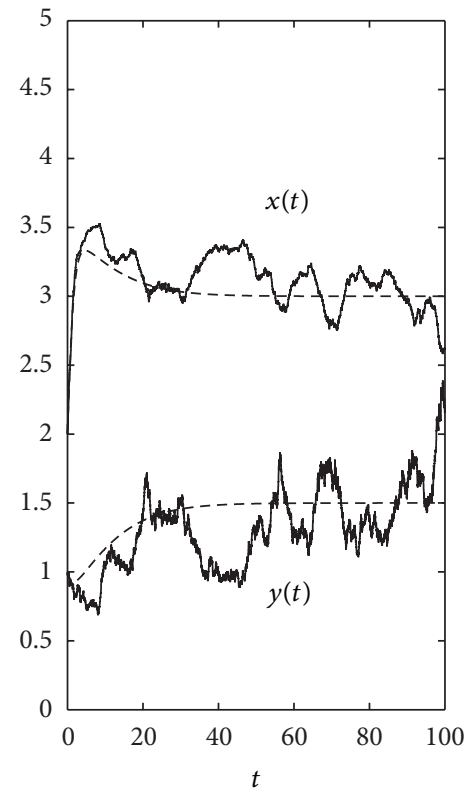

(b)

Figure 1: The solution of system (1) and system (3) with $(x(0), y(0))=(2,1), r=1.2, \mu=0.3, K=4, c=0.2, m c=0.1$. The red lines represent the solution of system (1), while the blue lines represent the solution of system (3) with $\sigma_{1}=0.01, \sigma_{2}=0.01$ in (a) and $\sigma_{1}=0.06$, $\sigma_{2}=0.08$ in (b), respectively.

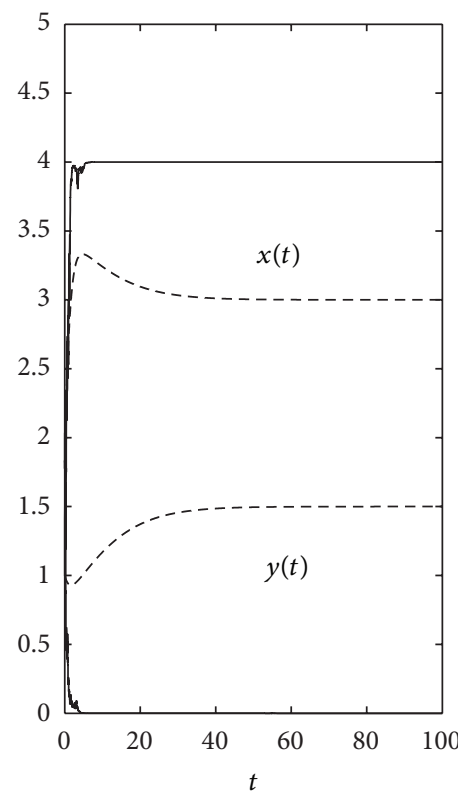

(a)

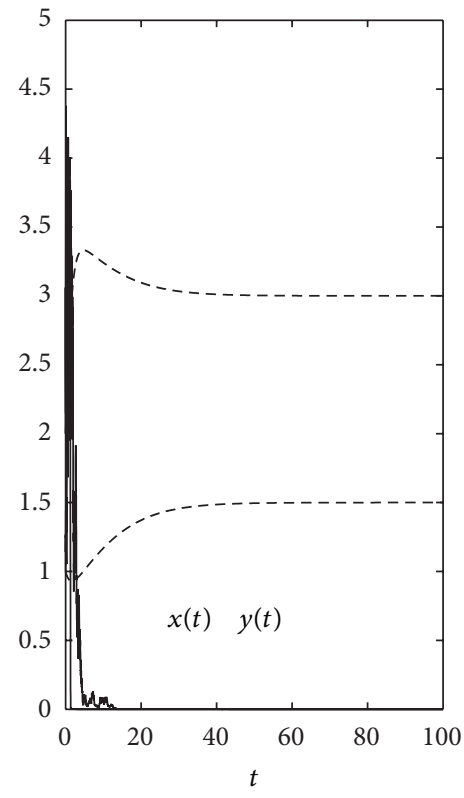

(b)

Figure 2: The solution of system (1) and system (3) with $(x(0), y(0))=(2,1), r=1.2, \mu=0.3, K=4, c=0.2, m c=0.1$. The red lines represent the solution of system (1), while the blue lines represent the solution of system (3) with $\sigma_{1}=1, \sigma_{2}=1$ in (a) and $\sigma_{1}=6, \sigma_{2}=1$ in (b), respectively. 
That is, for $\forall \epsilon>0$, there are constants $T_{0}$ and $\Omega_{\epsilon}$; then, if $t \geq T_{0}$ and $\omega \in \Omega_{\epsilon}$, we have $P\left(\Omega_{\epsilon}\right)>1-\epsilon$ and $y(t) \leq \epsilon$. So,

$$
\begin{aligned}
& x(t)\left(r-\frac{r}{K} x(t)-\epsilon\right) d t+\frac{r \sigma_{1}}{K}(K-x(t)) d B_{1}(t) \\
& \leq d x(t) \leq x(t)\left(r-\frac{r}{K} x(t)\right) d t+\frac{r \sigma_{1}}{K}(K-x(t)) d B_{1}(t),
\end{aligned}
$$

if $r>\sigma_{1}^{2} / 2$; from the arbitrariness of $\epsilon>0$, Lemma 2, and [23] (see Theorems 6.2 and 6.3), we could know that

$$
\lim _{t \rightarrow \infty} x(t)=K, \quad \text { a.s. }
$$

Concluding these arguments, we have the following theorem.

Theorem 12. Let $(x(t), y(t))$ be the solution of system (3) with any initial value $\left\{(x(0), y(0)) \in R_{+}^{2}, x(0) \in(0, K)\right\}$; then,

(1) if $r<\sigma_{1}^{2} / 2$, then

$$
P\left\{\lim _{t \rightarrow \infty} x(t)=0\right\} \geq \frac{\int_{Z(0)}^{+\infty} e^{\left(-2 / \sigma_{1}^{2}\right)\left(r-\sigma_{1}^{2} / 2\right) v}\left(1+e^{v}\right)^{-2} d v}{\int_{-\infty}^{+\infty} e^{\left(-2 / \sigma_{1}^{2}\right)\left(r-\sigma_{1}^{2} / 2\right) v}\left(1+e^{v}\right)^{-2} d v}
$$

where $Z(0)=\log (x(0) /(K-x(0)))$ and

$$
\begin{gathered}
\limsup _{t \rightarrow \infty} \frac{\log y(t)}{t}=-\mu-\frac{\sigma_{2}^{2}}{2}<0, \\
\omega \in \Omega_{0}=\left\{\omega: \lim _{t \rightarrow \infty} x(t)=0\right\} .
\end{gathered}
$$

That is to say, system (3) will be extinct in probability.

(2) If $\mu+\sigma_{2}^{2} / 2>m c K, r>\sigma_{2}^{2} / 2$, then

$$
\begin{aligned}
& \lim _{t \rightarrow \infty} y(t)=0 \quad \text { a.s., } \\
& \lim _{t \rightarrow \infty} x(t)=K, \quad \text { a.s. }
\end{aligned}
$$

\section{Numerical Simulation}

In this section, we give out the numerical experiment to support our results. Consider the equation

$$
\begin{gathered}
d x(t)=r x(t)\left(1-\frac{x(t)}{K}\right)-c x(t) y(t) \\
+\frac{\sigma_{1}}{K} x(t)(K-x(t)) d B_{1}(t), \\
d y(t)=-\mu y(t)+m c x(t) y(t)-\sigma_{2} y(t) d B_{2}(t) .
\end{gathered}
$$

By the Milstein method in [24], we have the difference equation

$$
\begin{aligned}
x_{k+1}=x_{k}+x_{k}[ & \left(r-\frac{r}{K} x_{k}-c y_{k}\right) \Delta t+\frac{\sigma_{1}}{K}\left(K-x_{k}\right) \epsilon_{1, k} \sqrt{\Delta} t \\
& \left.+\frac{\sigma_{1}^{2}}{2 K^{2}}\left(K-x_{k}\right)^{2}\left(\epsilon_{1, k}^{2} \Delta t-\Delta t\right)\right], \\
y_{k+1}=y_{k}+y_{k} & {\left[\left(-\mu+m c x_{k}\right) \Delta t\right.} \\
& \left.-\sigma_{2} \epsilon_{2, k} \sqrt{\Delta} t+\frac{\sigma_{2}^{2}}{2}\left(\epsilon_{2, k}^{2} \Delta t-\Delta t\right)\right],
\end{aligned}
$$

where $\epsilon_{1, k}$ and $\epsilon_{2, k}$ are the Gaussian random variables $N(0,1)$. By choosing $(x(0), y(0)) \in R_{+}^{2}$ and suitable parameters, by Matlab, we get Figures 1 and 2.

In Figure 1, choose parameters satisfying the condition of Theorem 9; system (3) is ergodic and the solution will persist in time average. Between picture (a) and (b), we only change the intensity parameters $\sigma_{1}$ and $\sigma_{2}$ and keep other parameters unchangeable. We observe that the amplitude of fluctuation is becoming large as the intensity of white noise is increasing. And we can see that the sample path is deviating from the corresponding deterministic system as the intensity of the white noise is becoming larger.

In Figure 2, we observe two cases. We observe case (1) in Theorem 12 and choose parameters such as $r>\sigma_{1}^{2} / 2, \mu+$ $\sigma_{2}^{2} / 2>m c K$ in (a); as Theorem 12 indicated, the prey will die out in probability and the predators will go to their carrying capacity. We also observe case (2) in Theorem 12 and choose parameters such as $r<\sigma_{1}^{2} / 2$ in (b); as Theorem 12 indicated, not only preys but also predators will die out in probability when the noise of the predators is large, and it does not happen in the deterministic system. This tells us strong environmental noise may cause species to become extinct. The larger the intensity environmental noise is, the bigger the probability of dying out is.

\section{Conflict of Interests}

The authors declare that there is no conflict of interests regarding the publication of this paper.

\section{Acknowledgments}

The work was supported by the Program for Changjiang Scholars and Innovative Research Team in University, NSFC of China, (no. 11371085), the Ph.D. Programs Foundation of Ministry of China (no. 200918), and the Natural Science Foundation of Jilin Province of China (no. 201115133). 


\section{References}

[1] T. C. Gard, "Stability for multispecies population models in random environments," Nonlinear Analysis: Theory, Methods \& Applications, vol. 10, no. 12, pp. 1411-1419, 1986.

[2] B. S. Goh, "Global stability in many species system," American Naturalist, vol. 111, pp. 135-143, 1997.

[3] J. L. Lv and K. Wang, "Asymptotic properties of a stochastic predator-prey system with Holling II functional response," Communications in Nonlinear Science and Numerical Simulation, vol. 16, no. 10, pp. 4037-4048, 2011.

[4] L. S. Chen and Z. J. Jing, "The existence and uniqueness of limit cycles for the differential equations of predator-prey interactions," Chinese Science Bulletin, vol. 9, pp. 521-523, 1984.

[5] X. R. Mao, G. Marion, and E. Renshaw, "Environmental Brownian noise suppresses explosions in population dynamics," Stochastic Processes and Their Applications, vol. 97, no. 1, pp. 95110, 2002.

[6] X. Mao, "Delay population dynamics and environmental noise," Stochastics and Dynamics, vol. 5, no. 2, article 149, 2005.

[7] P. Polansky, "Invariant distributions for multipopulation models in random environments," Theoretical Population Biology, vol. 16, no. 1, pp. 25-34, 1979.

[8] T. C. Gard, Introduction to Stochastic Differential Equations, Marcel Dekker, New York, NY, USA, 1988.

[9] Y. Kuang and H. L. Smith, "Global stability for infinite delay Lotka-Volterra type systems," Journal of Differential Equations, vol. 103, no. 2, pp. 221-246, 1993.

[10] Y. Z. Hu, F. K. Wu, and C. Huang, "Stochastic Lotka-Volterra models with multiple delays," Journal of Mathematical Analysis and Applications, vol. 375, no. 1, pp. 42-57, 2011.

[11] N. I. Wantanabe, Stochastic Differential Equations and Diffusion Processes, North-Holland, Amsterdam, The Netherlands, 1981.

[12] C. Y. Ji and D. Q. Jiang, "Dynamics of a stochastic density dependent predator-prey system with Beddington-DeAngelis functional response," Journal of Mathematical Analysis and Applications, vol. 381, no. 1, pp. 441-453, 2011.

[13] R. M. May, Stability and Complexity in Model Ecosystem, Princeton University Press, Princeton, NJ, USA, 2001.

[14] C. Y. Ji, D. Q. Jiang, and N. Z. Shi, "Analysis of a predator-prey model with modified Leslie-Gower and Holling-type II schemes with stochastic perturbation," Journal of Mathematical Analysis and Applications, vol. 359, no. 2, pp. 482-498, 2009.

[15] C. Y. Ji, D. Q. Jiang, and X. Y. Li, "Qualitative analysis of a stochastic ratio-dependent predator-prey system," Journal of Computational and Applied Mathematics, vol. 235, no. 5, pp. 1326-1341, 2011.

[16] X. N. Liu and L. S. Chen, "Complex dynamics of Holling type II Lotka-Volterra predator-prey system with impulsive perturbations on the predator," Chaos, Solitons \& Fractals, vol. 16, no. 2, pp. 311-320, 2003.

[17] X. R. Mao, Stochastic Differential Equations and Applications, Horwood, Chichester, UK, 1997.

[18] D.-Q. Jiang, B.-X. Zhang, D.-H. Wang, and N.-Z. Shi, "Existence, uniqueness, and global attractivity of positive solutions and MLE of the parameters to the logistic equation with random perturbation," Science in China A: Mathematics, vol. 50, no. 7, pp. 977-986, 2007.

[19] L. S. Chen and J. Chen, Nonlinear Biological Dynamical System, Science Press, Beijing, China, 1993.
[20] P. Y. Xia, X. K. Zheng, and D. Q. Jiang, "Persistence and nonpersistence of a nonautonomous stochastic mutualism system," Abstract and Applied Analysis, vol. 2013, Article ID 256249, 13 pages, 2013.

[21] D. Q. Jiang, C. Y. Ji, X. Y. Li, and D. O’Regan, "Analysis of autonomous Lotka-Volterra competition systems with random perturbation," Journal of Mathematical Analysis and Applications, vol. 390, no. 2, pp. 582-595, 2012.

[22] W. Feller, An Introduction to Probability Theory and Its Application, vol. 2, John Wiley \& Sons, New York, NY, USA, 1971.

[23] A. Gray, D. Greenhalgh, L. Hu, X. Mao, and J. Pan, "A stochastic differential equation SIS epidemic model," SIAM Journal on Applied Mathematics, vol. 71, no. 3, pp. 876-902, 2011.

[24] D. J. Higham, "An algorithmic introduction to numerical simulation of stochastic differential equations," SIAM Review, vol. 43, no. 3, pp. 525-546, 2001. 


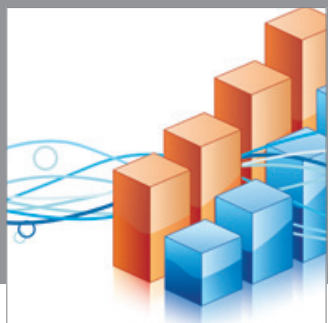

Advances in

Operations Research

mansans

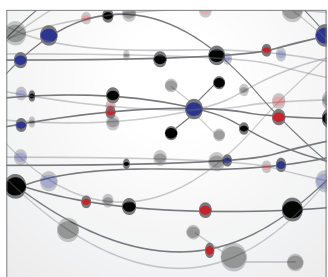

The Scientific World Journal
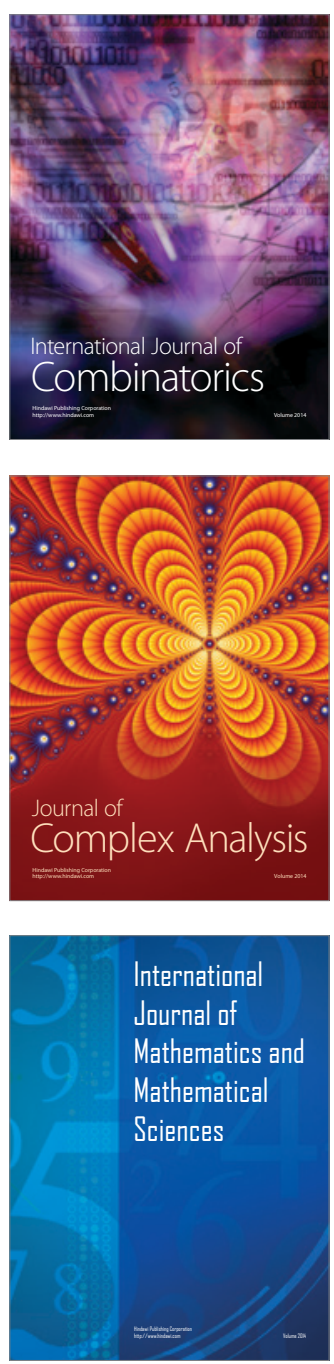
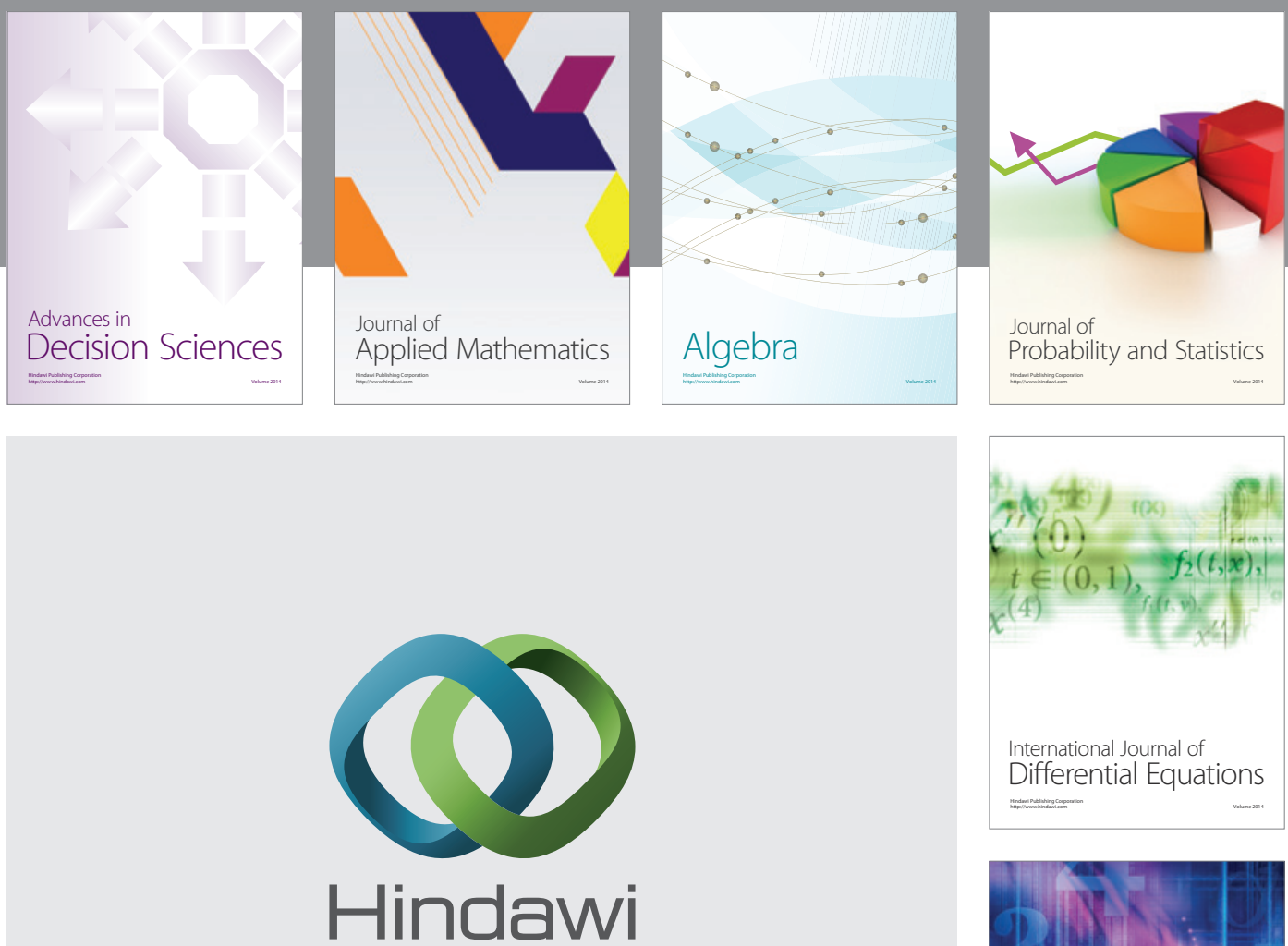

Submit your manuscripts at http://www.hindawi.com
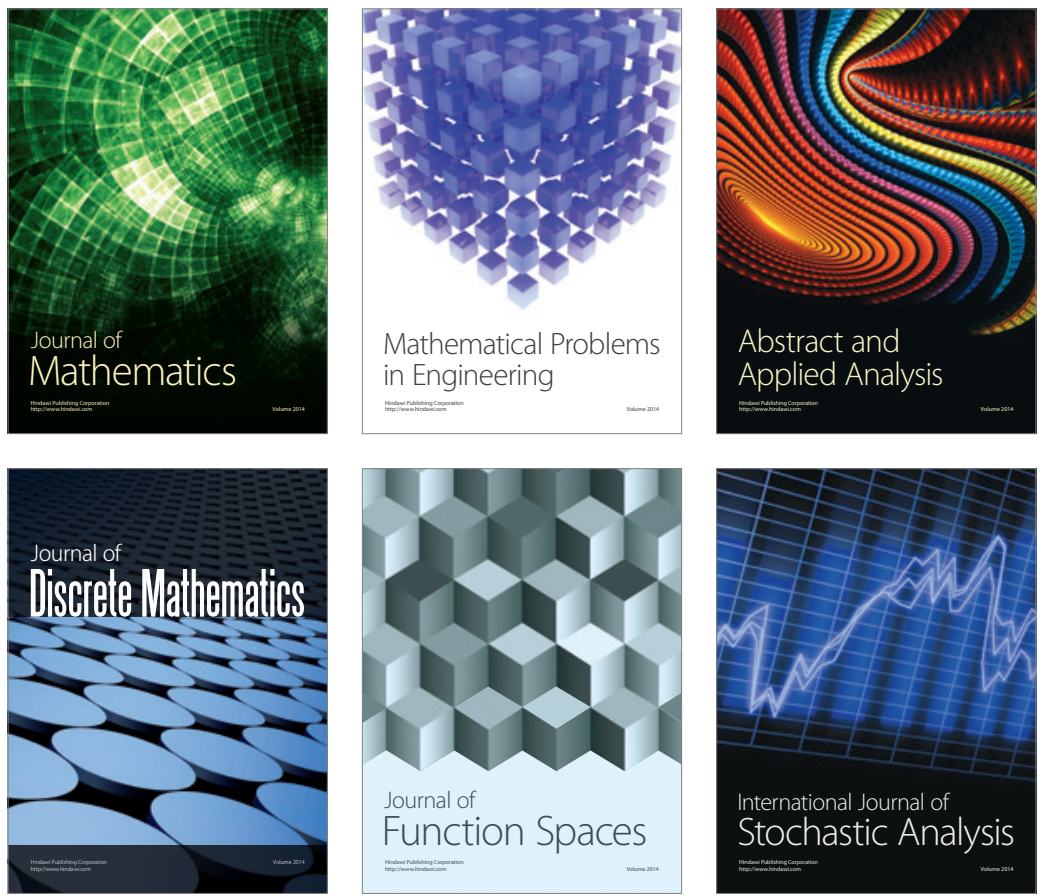

Journal of

Function Spaces

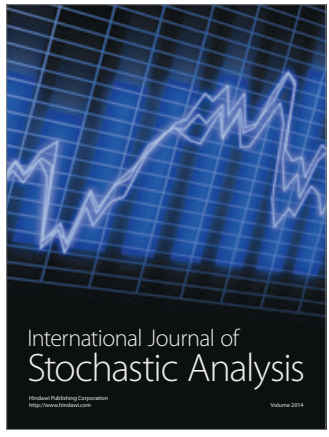

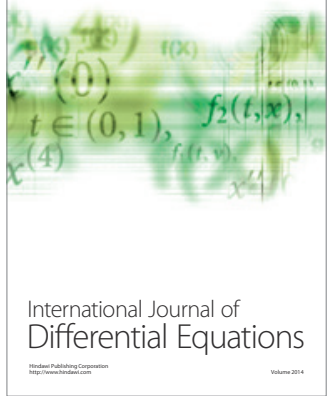
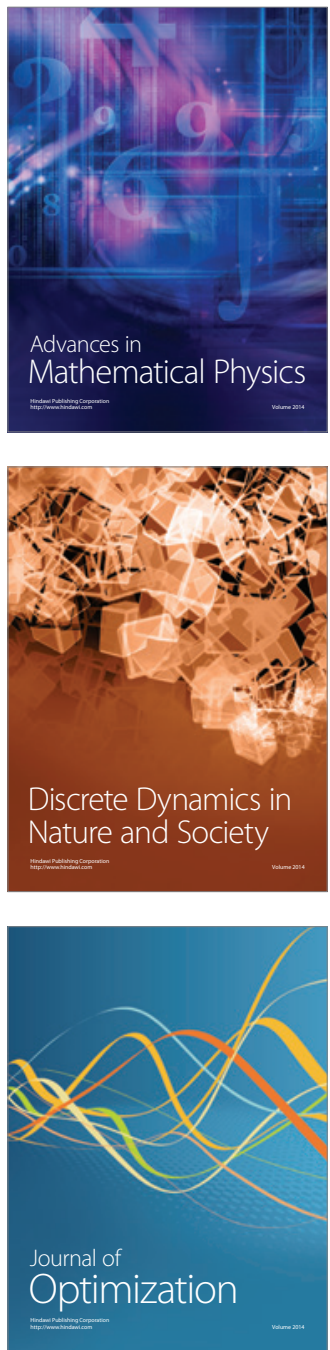